\title{
Characterization of novel immunodominant antigens of Mycobacterium tuberculosis
}

\author{
Rama Rao Amara, S. Shanti and Vijaya Satchidanandam
}

Department of Microbiology and Cell Biology, Indian Institute of Science, Bangalore 560012, India

\begin{abstract}
Author for correspondence: Vijaya Satchidanandam. Tel: +9180 3092685. Fax: +91803341683 or 3444697. e-mail: vijaya@cge.iisc.ernet.in

Seven novel antigens of Mycobacterium tuberculosis, which had previously been identified based on reactivity to sera from patients with tuberculosis, were characterized. Nucleotide sequence analysis of the genes encoding these seven antigens identified one of them as the FtsH and a second as the aminoimidazole ribotide synthase of $\boldsymbol{M}$. tuberculosis. Antisera raised to the recombinant forms of each of these seven antigens were used to study the distribution of these proteins within mycobacterial species as well as to determine their subcellular localization and hydrophobicity. Four of the seven antigens were conserved only among pathogenic strains of mycobacteria. Of the seven proteins studied, FtsH and a second protein of unknown identity were localized in membranes. Two were cytosolic, while two others, which had a high proline content, were tightly associated with the cell wall. One protein was secreted. This secreted protein could be identified by serum from a majority of tuberculosis patients but not BCG-vaccinated individuals, suggesting its potential use in the immunodiagnosis of tuberculosis.
\end{abstract}

Keywords: M. tuberculosis, tuberculosis patient sera, immunodiagnosis, FtsH

\section{INTRODUCTION}

Owing to the resurgence of tuberculosis, especially of multi-drug resistant (MDR) strains, there is today a greater need than ever before to identify antigens of Mycobacterium tuberculosis that can serve as immunodiagnostic agents and/or vaccine candidates. Several reports documenting the unique capability of live mycobacteria to protect mice from a challenge dose of the organism (Mackaness, 1967; Orme, 1988; Orme \& Collins, 1984; Rook et al., 1986) have suggested that bacteria actively replicating in vivo synthesize a set of antigens capable of eliciting protective immunity. Secreted antigens have been implicated as dominant members of this class by several investigators, an assumption largely validated by the impressive ability of purified culture filtrate antigens to protect mice and guinea pigs against a challenge with live virulent mycobacteria (Andersen, 1994; Horwitz et al., 1995; Pal \& Horwitz, 1992). To enhance the probability of identifying protective antigens unique to living mycobacteria, we screened a genomic DNA expression library of $M$. tuberculosis with sera from tuberculosis (TB) patients in advanced stage of the disease (Amara \& Satchidanandam, 1996). Preliminary nucleotide se-

Abbreviation: TB, tuberculosis. quence analysis revealed that the majority of these antigens were encoded by hitherto unreported genes of the organism. Interestingly, several of these antigens were able to elicit antibodies only when derived from live bacilli, but not when sonicated preparations of $M$. tuberculosis, which contained significant quantities of these same antigens (Amara \& Satchidanandam, 1997), were used to immunize mice and guinea pigs. In this communication we report further characterization of seven of these differentially immunogenic proteins of $M$. tuberculosis with respect to their subcellular localization, hydrophobicity, distribution among mycobacterial species and reactivity to sera from individual TB patients. The significance of these findings is discussed.

\section{METHODS}

Mycobacterial strains. M. tuberculosis strain NTI-83949 was isolated from the sputum of a patient with pulmonary tuberculosis and identified as reported previously (Amara \& Satchidanandam, 1996). All other mycobacterial strains were obtained from the Mycobacterial Repository Centre, Central Jalma Institute for Leprosy, Agra, India. Mycobacteria were grown in Middlebrook $7 \mathrm{H} 9$ medium supplemented with $0.5 \%$ albumin and $0.75 \%$ dextrose.

Serum samples. Serum samples were obtained from inmates of the Shantabai Devarao Shivaram Tuberculosis and Chest 
Diseases Hospital who had been suffering from TB for between one and ten months. Diagnosis of TB was based on presence of acid-fast bacilli in sputum and/or radiological findings. Antisera to the recombinant proteins were generated in Balb/c mice as follows. The recombinant protein band was excised from the Coomassie-blue-stained SDS-polyacrylamide gels following electrophoresis and ground into a paste. Protein emulsified with incomplete Freund's adjuvant was injected intraperitoneally into mice ( $10 \mu \mathrm{g}$ protein per mouse) at $15 \mathrm{~d}$ intervals twice followed by a third injection $20 \mathrm{~d}$ after the second using antigen suspended in PBS. Mice were bled $10 \mathrm{~d}$ later by intraocular puncture.

Protein analysis. Mycobacteria grown in Middlebrook $7 \mathrm{H} 9$ broth were harvested in mid-exponential phase and washed once in ice-cold sterile PBS. The cell pellet was resuspended in ice-cold water containing protease inhibitors $(0.2 \mathrm{mM}$ PMSF and $2.5 \mu \mathrm{g} \mathrm{ml}^{-1}$ each of aprotinin, antipain, leupeptin, pepstatin and chymostatin). The bacillary suspension was sonicated for a total of 15 min at setting 3 in a Branson model 450 sonifier. The sonicate was dried in a Savant Speedvac concentrator and resuspended in lysis buffer $(10 \mathrm{mM}$ sodium phosphate, $\mathrm{pH} 9.0,6 \mathrm{M}$ urea, $1 \% \mathrm{SDS}, 1 \% \beta$-mercaptoethanol) at a concentration of $5-10 \mathrm{mg} \mathrm{ml}^{-1}$. Approximately $50 \mu \mathrm{g}$ protein was electrophoresed per lane of an SDS polyacrylamide gel, transferred to nitrocellulose membrane (Schleicher and Schuell, BA85) using a homemade semi-dry transfer unit for $1 \mathrm{~h}$ at $1.5 \mathrm{~mA} \mathrm{~cm}^{-2}$ and probed for $3 \mathrm{~h}$ with pooled mouse or human primary antibody, followed by treatment with appropriate horseradish-peroxidaseconjugated secondary antibody. Bands were visualized by incubating with the substrate diaminobenzidine dihydrochloride and hydrogen peroxide. Western blot analysis of recombinant Escherichia coli SOLR lysates utilized sufficient total protein to contain $100-200 \mathrm{ng}$ specific recombinant protein as judged from Coomassie-blue-stained gels.

Southern blot analysis. Genomic DNA was isolated from mycobacteria grown in $7 \mathrm{H} 9$ broth as described previously (Amara \& Satchidanandam, 1996), digested with EcoRI and SalI to completion and electrophoresed on $0.8 \%$ agarose gels. DNA transferred to nylon membranes was hybridized to specific fragments obtained from the respective gene sequences, labelled using the Gene Images-CDP detection kit from Amersham Life Science. The probe used for ARRMTB11 was a $600 \mathrm{nt} A v a \mathrm{I}$ fragment from the middle of the gene; that for clone 73 was a 700 nt EcoRI-SalI fragment encompassing the entire gene; that for clone 268 was a $250 \mathrm{nt}$ EcoRI-KspI fragment; that for clone 283 was a $500 \mathrm{nt}$ EcoRI-BstXI fragment. Care was taken to ensure that all the probes contained sequences solely from within the open reading frames (ORFs) specifying the recombinant proteins of interest.

DNA sequencing. The recombinant pBluescript plasmids were sequenced using the T3 primer in a ABI Prism automated sequencing system using Taq DNA polymerase.

Subcellular fractionation. Sonicated preparations of $M$. tuberculosis were centrifuged at $19000 \mathrm{~g}$ for $30 \mathrm{~min}$ at $4^{\circ} \mathrm{C}$ and the pellet consisting of the cell wall fraction was kept aside. The clear supernatant was centrifuged at $100000 \mathrm{~g}$ for $60 \mathrm{~min}$ at $4^{\circ} \mathrm{C}$ to precipitate the membranes, and the supernatant consisting of cytosolic proteins was dried in a Savant Speedvac concentrator. All fractions were resuspended in lysis buffer and an amount of protein equivalent to $300 \mu \mathrm{l}$ culture was electrophoresed per lane of an SDS-polyacrylamide gel $(10 \%$, $\mathrm{w} / \mathrm{v}$, acrylamide) followed by Western blotting to sera raised in mice to each of the recombinant proteins.
Triton X-114 phase separation of sonicate proteins. Phase separation in the presence of Triton X-114 was carried out essentially as described by Bordier (1981). Supernatants from M. tuberculosis sonicate devoid of cell walls at a concentration of $3 \mathrm{mg} \mathrm{ml}^{-1}$ were brought to a concentration of $50 \mathrm{mM}$ and $150 \mathrm{mM}$ of Tris, $\mathrm{pH} 8.0$, and $\mathrm{NaCl}$, respectively, followed by Triton X-114 to a final concentration of $3 \%$. This solution was maintained for $30 \mathrm{~min}$ on ice with frequent vortexing. The sample was then incubated at $37^{\circ} \mathrm{C}$ for $5 \mathrm{~min}$ followed by centrifugation at $5000 \mathrm{~g}$ for $5 \mathrm{~min}$ at room temperature. The upper aqueous and lower detergent phases were both reextracted once. Proteins from the detergent phase were recovered by acetone precipitation at $-20^{\circ} \mathrm{C}$ overnight, dissolved in lysis buffer and used for Western blot analysis.

\section{RESULTS}

\section{Analysis of nucleotide and protein sequences of novel genes}

We had earlier reported the differential immunogenicity of several novel antigens of $M$. tuberculosis derived from live versus dead bacilli (Amara \& Satchidanandam, 1997). The partial nucleotide sequences at the beginning of the inserts encoding seven among these recombinants, represented by clones ARRMTB-2, 11, 14, 39, 73, 268 and 283 (accession numbers and protein sizes listed in Amara \& Satchidanandam, 1997) from the lambda ZAP II:: M. tuberculosis genomic DNA library, were compared to all entries in the GenBank and EMBL databases and the M. tuberculosis sequence database at the Sanger Centre, Hinxton, Cambridge, UK. The nucleotide sequence of clone 2 showed a perfect match to an ORF within cosmid MTCY7H7B in the $M$. tuberculosis cosmid database which was found to be homologous to the cell division protein FtsH from several species. Percentage identities ranged from 44 to 53 when the BLAST algorithm was used for comparison. The size of $82 \mathrm{kDa}$ for $M$. tuberculosis FtsH (760 amino acids) is significantly larger than that of other bacteria $(644$ and 637 amino acids for $E$. coli and Bacillus subtilis $\mathrm{FtsH}$ respectively) and similar to FtsH of yeast (761 amino acids). Similarly, the sequence of clone 14 , which was identical to an ORF in the contig TB452 at the Sanger database, showed $84 \%$ identity to aminoimidazole ribotide synthase of $M$. leprae (GenBank accession no. U15182) and identities ranging from 52 to $58 \%$ to aminoimidazole ribotide synthases from other bacteria, plant and mammals in a BLAST analysis. The sequence of clone 73 showed low-level similarity (identities ranging from 23 to $31 \%$ in a BLAST analysis) to enoyl-CoA hydratase, carnitine racemase and naphthoate synthase but differed considerably from that of the putative enoyl-CoA hydratase of $M$. tuberculosis (EMBL accession no. Q10533).

The translated amino acid sequences of clones 11, 39, 268 and 283 had no homology to any known proteins. We found significant similarity between clones 268 and 283 in the nucleotide and translated amino acid sequences, although antibodies raised to these two recombinant proteins showed no cross-reactivity. This may be due to unique stretches downstream of the regions sequenced by us, which fall in the C-terminal 


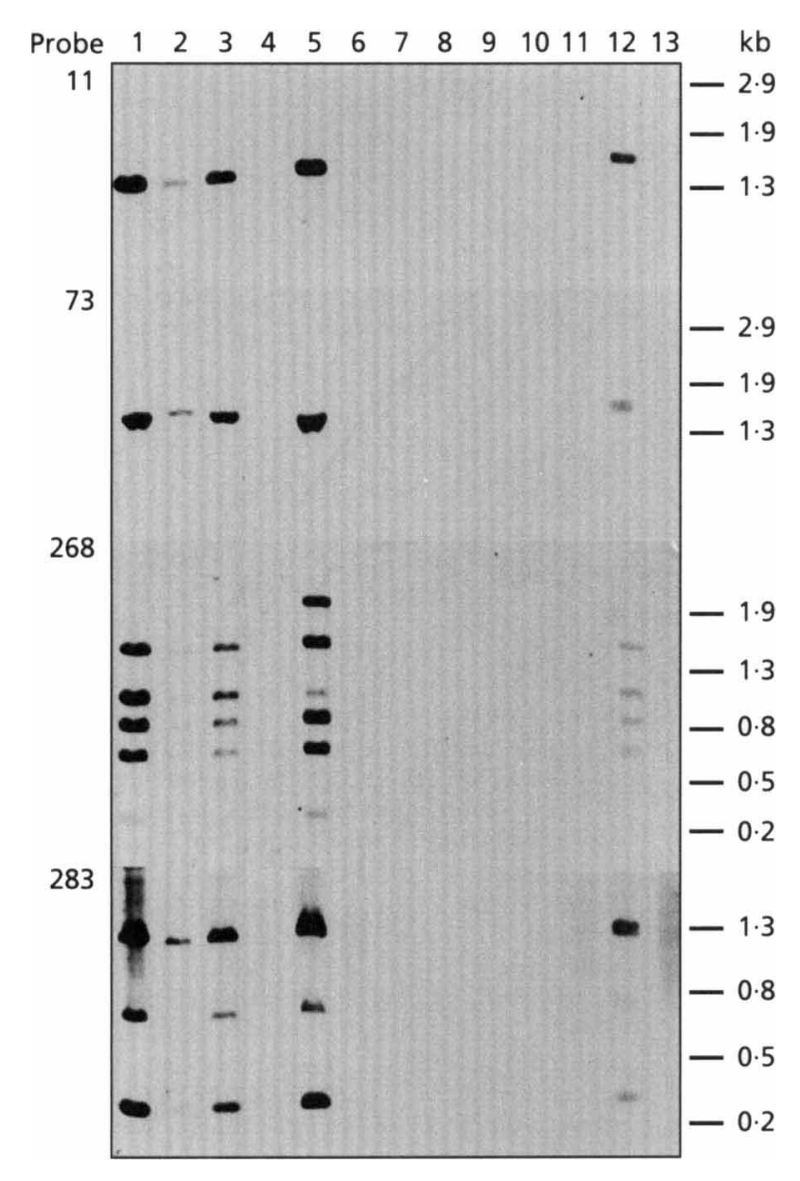

Fig. 1. Distribution of novel genes in mycobacteria. DNA $(10$ $\mu \mathrm{g}$ ) isolated from each mycobacterium was digested with EcoRI and Sall and electrophoresed in $0.8 \%$ agarose gels. DNA transferred to a nylon membrane was hybridized to the probes derived from the recombinant clones labelled using the Gene Images-CDP detection kit (Amersham). Numbers on the left of each panel refer to clone numbers from which probes were derived. Positions of molecular size markers are indicated. Lanes: 1, $M$. tuberculosis 64719; 2, $M$. tuberculosis H37Ra; 3, $M$. tuberculosis H37Rv; $4, M$. simiae; $5, M$. bovis; $6, M$. scrofulaceum; 7, M. intracellulare; 8, $M$. szulgai; 9, M. kansasii; $10, M$. fortuitum; 11, M. microti; $12, M$. bovis-BCG; $13, M$. vaccae.

ends of the specified proteins, since our recombinants represent $\mathrm{N}$-terminally truncated mycobacterial proteins fused to the polypeptide specified by the pBSK vector sequences. In fact, antibodies raised to recombinant 283 identified two proteins in lysates of $M$. tuberculosis, of 38 and $84 \mathrm{kDa}$, while those raised to recombinant 268 identified a single protein of $\sim 95 \mathrm{kDa}$ (Amara \& Satchidanandam, 1997).

Southern hybridization of the inserts from clones 268 and 283 to genomic DNA of M. tuberculosis revealed four bands strongly hybridizing to the $250 \mathrm{bp} 268$ probe and three bands hybridizing to the $500 \mathrm{bp} 283$ probe, two of which (of 300 and $800 \mathrm{nt}$ ) were also present in common with the 268 probe (Fig. 1 probe 268, lanes 1-3). A search of the M. tuberculosis sequence database generated by the Sanger Centre for stretches hom- ologous to 268 and 283 identified the cosmid MTCY49 as being homologous to both clones. These sequences were also present in the cosmid MTAD8 submitted to the EBI database by Genome Therapeutics Corporation, which has no overlap with MTCY49. The predicted polypeptide sequences of the two ORFs (MTCY49.21 and MTAD8.13) show $54.4 \%$ identity. This suggests that a segment of the 268 gene may be present in more than one copy in the M. tuberculosis genome. Sequences hybridizing to these two genes were also found in $M$. bovis and BCG (Fig. 1, probes 268 and 283, lanes 5 and 12). In contrast to 268 and 283 , Southern hybridization of M. tuberculosis genomic DNA to the probes from clones 11 and 73 identified unique bands hybridizing to each of these two probes (Fig. 1, probes 11 and 73, lanes 1-3). All four above-mentioned genes also hybridized to genomic DNA from M. bovis and M. bovis BCG (Fig. 1, lanes 5 and 12). In contrast to this restricted distribution, probes derived from clones 2 and 39 hybridized to genomic DNA from all the mycobacterial strains tested, while that from 14 hybridized to all except $M$. fortuitum, M. scrofulaceum, M. intracellulare and M. szulgai (data not shown).

\section{Distribution of the proteins among mycobacteria}

We next looked for the presence of the proteins encoded by each of these seven genes in other species of mycobacteria using antibodies raised in mice to the recombinant proteins. While proteins 2 and 39 were widely distributed in all the mycobacterial species tested, antibodies raised to protein 14 cross-reacted with similar-sized proteins in most pathogenic and nonpathogenic mycobacteria tested (Fig. 2a). Antibodies raised to recombinant 39 identified, in addition to the major species of $43 \mathrm{kDa}$, a second, larger protein weakly in a few mycobacteria. The proteins 73,268 and 283 had a restricted distribution in some of the pathogenic mycobacteria (Fig. 2b): protein 73 was present in $M$. bovis, M. bovis BCG and M. kansasii in addition to $M$. tuberculosis while protein 268 was present also in $M$. szulgai in addition to the aforementioned three species. This result for proteins 73 and 268 was in contrast to the data obtained by Southern hybridization (Fig. 1, probes 11 and 73, lanes 8 and 9); differences in codon usage between $M$. kansasii and $M$. szulgai perhaps resulted in lack of hybridization of the 73 and 268 probes to these genomes. Protein 283 was found only in M. tuberculosis, $M$. bovis and BCG, which was corroborated by the DNA hybridization data, while that specified by clone 11 appeared unique to $M$. tuberculosis. This was in contrast to the data obtained in Southern hybridization, which revealed the presence of sequences in $M$. bovis and $M$. bovis BCG which also hybridized to clone 11 (Fig. 1, probe 11, lanes 5 and 12).

\section{Subcellular localization}

Lysates of bacilli were separated into cell wall, membrane and cytoplasmic fractions, as described in Methods. These fractions were then analysed for the 

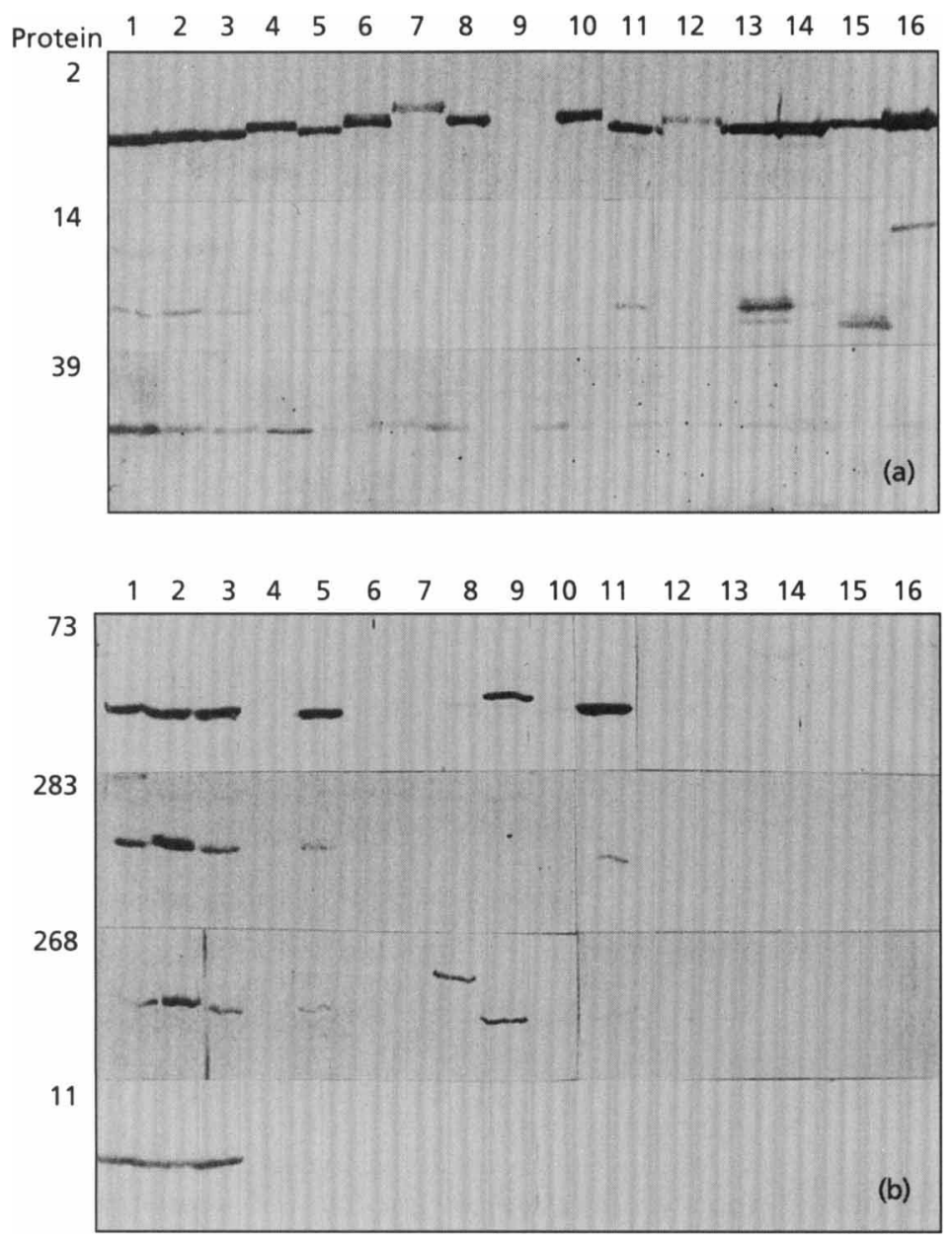

Fig. 2. Distribution of antigens in mycobacteria. Mouse serum raised to each of the seven recombinant proteins was immunoblotted to sonicated antigen preparations obtained from several mycobacteria. (a) Widely distributed proteins 2, 14 and 39 ; (b) proteins 73, 283, 268 and 11, with more restricted distribution. Lanes: $1, M$. tuberculosis NTI83949; 2, $M$. tuberculosis H37Ra; 3, M. tuberculosis H37Rv; 4, M. simiae; 5, $M$ bovis; $6, M$. scrofulaceum; $7, M$. intracellulare; 8. M. szulgai; 9, M. kansasii; 10, $M$. fortuitum; 11, $M$. bovis BCG; $12, M$. parafortuitum; 13, M. phlei; 14, $M$. vaccae; $15, M$. flavescens; $16, M$. chitae. For clone 2 , $M$. kansasii was not included.
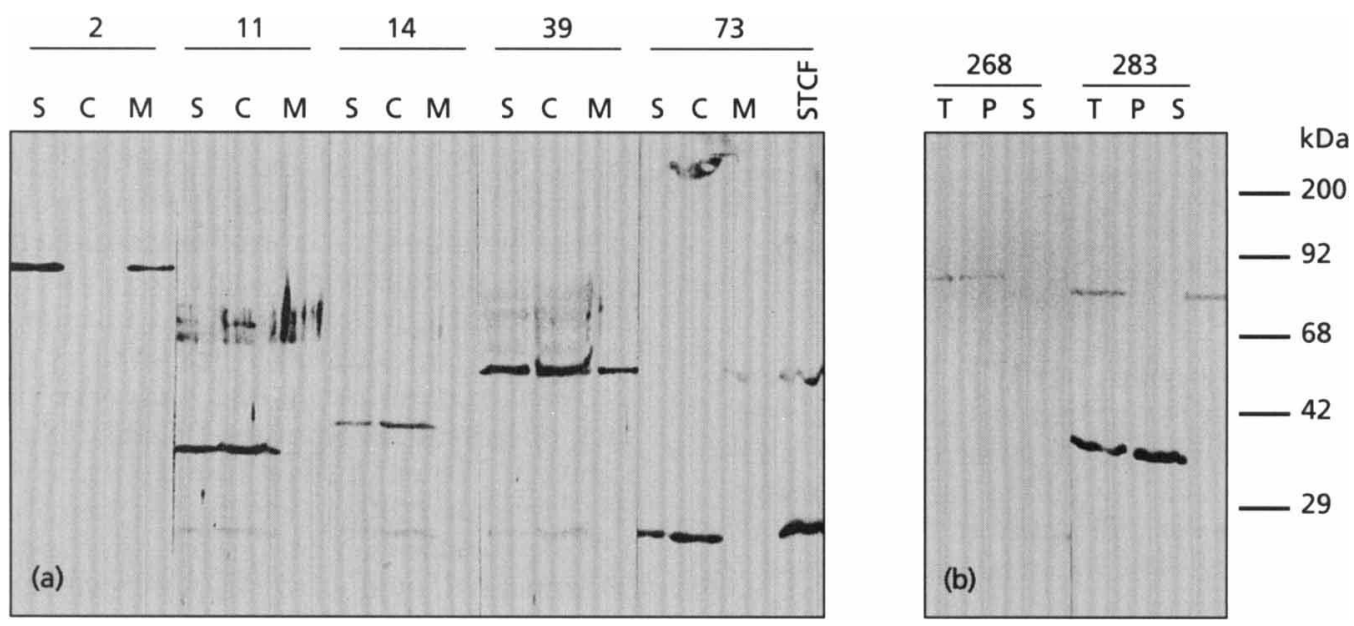

Fig. 3. (a) Subcellular localization of $M$. tuberculosis proteins. Sonicated preparations of mycobacteria were fractionated into cell walls, membranes and cytosol, as described in Methods. The fractions were Western blotted to mouse serum raised to individual recombinant proteins from the clones whose numbers are indicated at the top of the figure. $S$, soluble supernatant fraction devoid of unlysed cells and cell walls; C, cytosol; M, membranes; STCF, short-term culture filtrate. (b) Total sonicated preparations of mycobacteria ( $T$ ) were centrifuged at $15000 \mathrm{~g}$ to obtain the pellet $(P)$ and supernatant fractions (S). Fractions were Western blotted as in (a). Positions of molecular mass markers are indicated. 


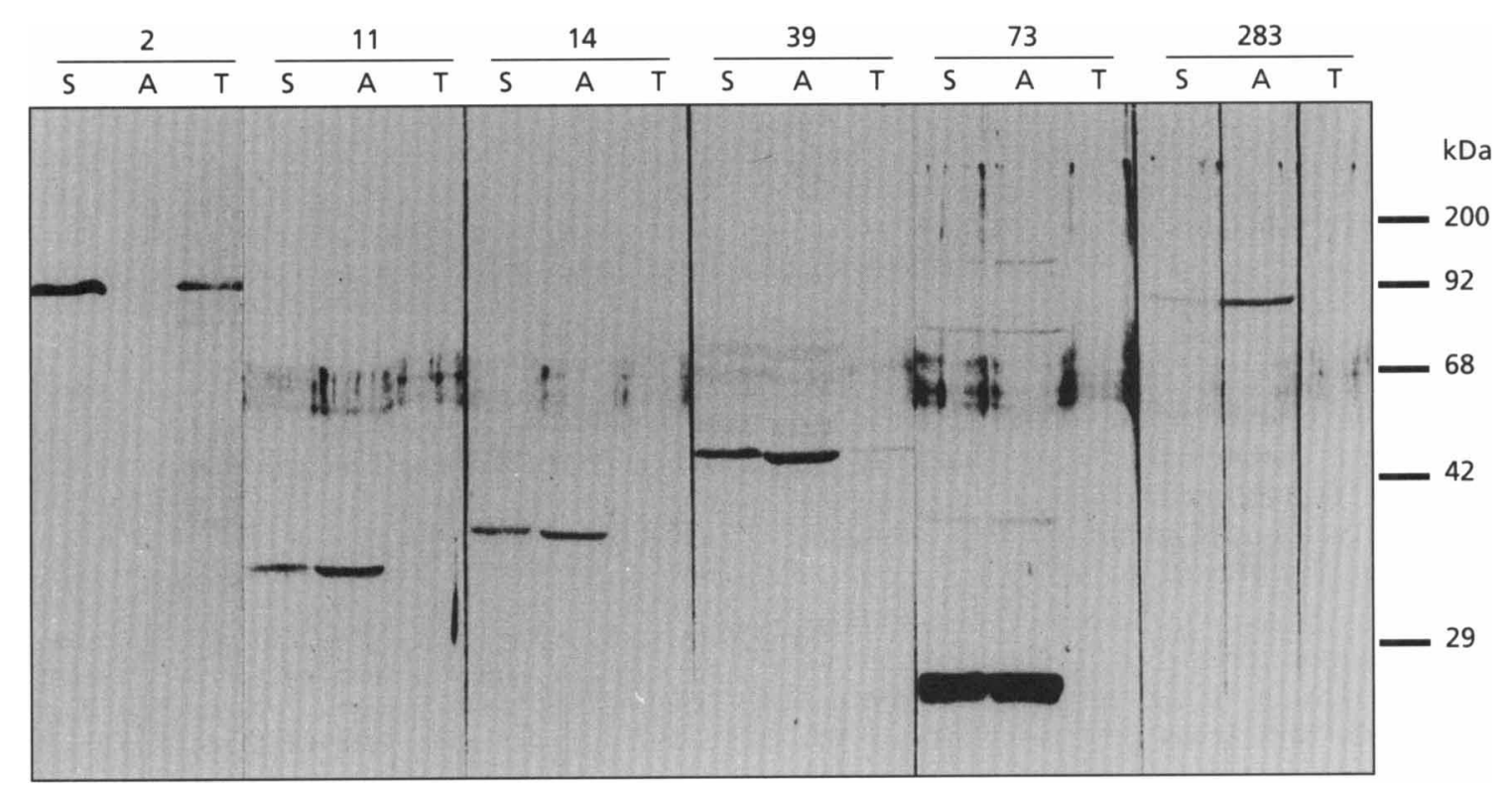

Fig. 4. Triton X-114 phase separation of $M$. tuberculosis proteins. Soluble proteins of $M$. tuberculosis free of cell walls were subjected to Triton X-114 phase separation, as described in Methods. The fractions were Western blotted to mouse serum raised to individual recombinant proteins from the clones whose numbers are indicated at the top of the figure. $S$, soluble fraction; A, aqueous phase; T, Triton X-114 phase. Positions of molecular mass markers are indicated.

presence of the proteins corresponding to each of the seven clones. Protein 268 and the smaller protein of 283 were exclusively present in the cell wall fraction (Fig. $3 \mathrm{~b})$, resulting in their absence in the sonicate supernatant that was used in the fractionation experiments depicted in Fig. 3(a). Proteins 11, 14 and 73 were exclusively present in the cytosolic fraction. Protein 2 (FtsH) was present in the membrane fraction, a location expected for this cell division protein, while protein 39 was found distributed between the cytosol and the membrane fraction (Fig. 3a). The larger 283 protein was found in the cytosol fraction (data not shown).

\section{Triton X-114 phase separation}

To study the hydrophobic nature of these antigens, we analysed their ability to partition into Triton X-114. Proteins corresponding to clones 11, 14, 73 and the larger protein of 283 partitioned completely in the aqueous phase, indicating their hydrophilic nature (Fig. 4). Protein 2 (FtsH) was present exclusively in the detergent phase, consistent with its subcellular localization in membranes. The protein corresponding to clone 39 was distributed between the aqueous and Triton X-114 phases.

\section{Reactivity to sera from TB patients}

The immunodominance of these antigens during the active phase of disease prompted us to explore the possibility of using them as diagnostic reagents. Since the proteins reported here were originally identified using sera from a pool of 13 TB patients (Amara \& Satchidanandam, 1996), we examined the reactivity of individual serum samples to the recombinant antigens under study. Recombinants 73 and 14 were identified by a majority of the sera tested. All 12 sera from chronically infected TB patients as well as 22 out of 29 serum samples of patients in the early phase of the disease showed reactivity to 73 (data not shown). No antibodies were detected to recombinant 73 in sera from two healthy contacts or a PPD-negative individual. The presence of antibodies to recombinant 73 alone in patients suggests its potential use in immunodiagnosis of TB.

\section{DISCUSSION}

The genes for all the proteins characterized in this report were originally identified as those encoding proteins reactive to sera from TB patients (Amara \& Satchidanandam, 1996). To our knowledge, this represented the first attempt to identify a large number of immunodominant antigens of $M$. tuberculosis reactive to a pool of TB patient sera. Previous attempts at screening expression libraries of this organism had utilized monoclonal or polyclonal sera raised to preparations of killed M. tuberculosis; several antigens thus identified were subsequently found to react to sera from TB patients. This perhaps explains the novel nature of the genes we identified, an assumption strengthened by our recent report (Amara \& Satchidanandam, 1997) documenting the differential immunogenicity of antigens derived from live versus dead tubercle bacilli. Several researchers have observed and reported differences in immunogenicity and protective ability between live and killed tubercle bacilli (Mackaness, 1967; Orme, 1988; Orme \& Collins, 1984; Rook et al., 1986). Antigens 
secreted into the culture fluid by M. tuberculosis have been invoked as major contributors to the superior protective efficacy of live tubercle bacilli. In support of this, total secreted antigen preparation of $M$. tuberculosis was shown to protect both guinea pigs and mice from a subsequent challenge with live $M$. tuberculosis (Andersen, 1994; Horwitz et al., 1995; Pal \& Horwitz, 1992). The seven immunodominant antigens characterized in this report, however, showed a wide distribution between the cytosol, membranes and cell walls of the organism. We found that the protein specified by clone 73 alone was secreted by the organism $(R$. R. Amara and others, unpublished results), indicating that proteins localized within the bacilli are also immunodominant during active disease.

A protein matching the size and distribution we found for antigen 73 was reported earlier (Rambukkana et al., 1993) in culture filtrates of M. tuberculosis; the relationship between these two proteins warrants investigation. Although the sequence of antigen 73 showed reasonable similarity to enzymes of fatty acid metabolism, this level of similarity was much less than that observed between the multiple entries for each of these enzymes in the database. The presence of antigen 73 in culture supernatants was unexpected for a protein with homology to enzymes of fatty acid metabolism. However, this is reminiscent of a recent report (Belisle et al., 1997) showing that the dominant secreted proteins of the antigen 85 complex of $M$. tuberculosis have in vitro mycolyltransferase activity, suggesting that they may be enzymes involved in mycobacterial cell wall biosynthesis. It is likely therefore that the protein specified by clone 73 may also be an enzyme involved in cell wall biogenesis.

Two of the seven proteins under study, identified as FtsH and aminoimidazole ribotide synthase, are essential for active replication. Two other proline-rich proteins (268 and 283) were localized in the cell wall, which in the case of $M$. tuberculosis is a highly specialized structure implicated in the unusually high resistance of these bacilli to several antibiotics and chemotherapeutic agents. These two proline-rich proteins may play an important role in maintaining the structure of the mycobacterial cell wall. Interestingly, these proteins were specific only to pathogenic mycobacteria. The only protein among the seven that was unique to $M$. tuberculosis was that specified by clone 11 . However, we observed sequences in the genomic DNA obtained from $M$. bovis and BCG that hybridized to clone 11. This may be explained by the fact that the antibodies used in the Western blotting experiments were raised to the C-terminal $8 \mathrm{kDa}$ stretch of the protein (which may be unique to $M$. tuberculosis) while the DNA segment used in the Southern hybridization was derived from sequences further upstream in the gene, where the sequences homologous to M. bovis may have been present.

Immunodominant proteins with a limited distribution among pathogenic mycobacteria would be the best candidates for use in immunodiagnosis of TB. We found that proteins specified by clones 73 and 14 were identified by a large proportion of individual patient sera. The absence of antibodies to 73 in BCG-vaccinated and PPDnegative individuals further adds to the promise of this antigen as a potential diagnostic agent. The utility of these antigens in immunodiagnosis can only be confirmed after more exhaustive serological studies.

\section{ACKNOWLEDGEMENTS}

Our thanks are due to Sujatha Chandrasekharan and Vijay Czhallu of the National Tuberculosis Institute. We thank the physicians and staff of the Shantabai Devarao Shivaram Tuberculosis and Chest Diseases Hospital. We thank V. M. Katoch, of the Central Jalma Institute for Leprosy, for providing the various strains of mycobacteria used in this study. We acknowledge the excellent technical assistance of M. S. Sudha. This work was supported by a grant (BT/R\&$\mathrm{D} / 15 / 12 / 92$ ) to $\mathrm{S}$. V. from the Department of Biotechnology, Government of India. A.R.R. is the recipient of a senior research fellowship from the Council for Scientific and Industrial Research.

\section{REFERENCES}

Amara, R. R. \& Satchidanandam, V. (1996). Analysis of a genomic DNA expression library of Mycobacterium tuberculosis using tuberculosis patient sera: evidence for modulation of host immune response. Infect Immun 64, 3765-3771.

Amara, R. R. \& Satchidanandam, V. (1997). Differential immunogenicity of novel Mycobacterium tuberculosis antigens derived from live versus dead bacilli. Infect Immun 65, 4880-4882.

Andersen, P. (1994). Effective vaccination of mice against Mycobacterium tuberculosis infection with a soluble mixture of secreted mycobacterial proteins. Infect Immun 62, 2536-2544.

Belisle, J. T., Varalakshmi, D. V., Sievert, T., Takayama, K., Brennan, P. J. \& Besra, G. S. (1997). Role of the major antigen of Mycobacterium tuberculosis in cell wall biogenesis. Science 276, 1420-1422.

Bordier, C. (1981). Phase separation of integral membrane proteins in Triton X-114 solution. J Biol Chem 256, 1604-1607.

Horwitz, M. A., Lee, B.-W. E., Dillon, B. J. \& Harth, G. (1995). Protective immunity against tuberculosis induced by vaccination with major extracellular proteins of $M y$ cobacterium tuberculosis. Proc Natl Acad Sci USA 92, 1530-1534.

Mackaness, G. B. (1967). The relationship of delayed hypersensitivity to acquired cellular resistance. $\mathrm{Br}$ Med Bull 23, 52-54.

Orme, I. M. (1988). Induction of nonspecific acquired resistance and delayed-type hypersensitivity, but not specific acquired resistance, in mice inoculated with killed mycobacterial vaccines. Infect Immun 56, 3310-3312.

Orme, I. M. \& Collins, F. M. (1984). Adoptive protection of the Mycobacterium tuberculosis-infected lung. Dissociation between cells that passively transfer protective immunity and those that transfer delayed-type hypersensitivity to tuberculin. Cell Immunol 84, 113-120.

Pal, P. G. \& Horwitz, M. A. (1992). Immunization with extracellular proteins of Mycobacterium tuberculosis induces cellmediated immune responses and substantial protective immunity in a guinea pig model of pulmonary tuberculosis. Infect Immun 60, 4781-4792.

Rambukkana, A., Das, P. K., Kolk, A. H. J., Burggraff, J. D., 
Kuijper, S. \& Harboe, M. (1993). Identification of a novel $27-\mathrm{kDa}$ protein from $M$. tuberculosis culture fluid by a monoclonal antibody specific for the $M$. tuberculosis complex. Scand $J$ Immunol 37, 471-478.

Rook, G. A. W., Steele, J., Barnass, S., Mace, J. \& Stanford, J. L. (1986). Responsiveness to live $M$. tuberculosis, and common antigens, of sonicate-stimulated $\mathrm{T}$ cell lines from normal donors. Clin Exp Immunol 63, 105-110.

Received 14 October 1997; revised 15 January 1998; accepted 19 January 1998. 